Erschienen in: Stickel, Gerhard (Hrsg.): Varietäten des Deutschen. Regional- und Umgangssprachen. - Berlin, New York: de Gruyter, 1997. S. 392-396. (Institut für deutsche Sprache. Jahrbuch 1996)

\title{
INGO REIFFENSTEIN
}

\section{Dialektverfall oder Mundartrenaissance? - in Bayern und Österreich}

Die im Thema gestellte Alternative stellt sich nach meiner Einschätzung für den Südosten des deutschen Sprachgebietes nicht. Wenn man den Begriff "Verfall" wörtlich nimmt, würde das auf den bevorstehenden oder eingetretenen Sprachtod der Varietät Dialekt hinauslaufen. Davon 
aber in Bayern und in Österreich keine Rede sein. Die Dialekte leben, in wie veränderten Formen und z.T. Funktionen auch immer. Und da die Dialekte nicht gestorben sind, bedürfen sie auch keiner Renaissance, d.h. Wiedergeburt. Verändert hat sich in den letzten 50 Jahren natürlich viel, und da gibt es auch im Süden erhebliche regionale Verschiedenheiten. Was fast überall beobachtet werden kann, das ist Dialektabbau, d.h. die Ersetzung altartiger Dialektmerkmale durch jeweils standardsprachnähere Formen. Gegen das von Arno Ruoff (in diesem Band S. 142ff.) für den deutschsprachigen Südwesten gezeichnete Bild halte ich für das Bayerisch-Österreichische fest, daß altartige, kleinräumig verteilte Dialektmerkmale auf allen Ebenen (Phonologie, Morphologie, Lexikon und Syntax) unter dem Druck stehen, durch prestigebesetzte, großräumiger verbreitete Merkmale ersetzt zu werden. Die Richtung dieser Veränderungen geht dabei immer zu umgangssprachenäheren $\mathrm{Va}$ rietäten, von den kleinräumigen altartigen Dialekten zu groBräumigeren Dialekt- und Umgangssprache-Gebieten. Der Abstand der neuen Dialekte zur Standardsprache ist immer geringer als jener der Altdialekte. Solche Ab- und Umbauprozesse können aber nicht als Verfall bezeichnet werden, sondern sie sind nichts anderes als Phänomene des Sprachwandels. Richtig ist, daB Vorgänge dieser Art in den letzten $50 \mathrm{Jahren}$ rascher verlaufen sind als in den Jahrhunderten zuvor.

Die aus den Veränderungen resultierenden Umgangssprachen sind immer regional gebunden, $d$. $h$. dialektal determiniert. Die Unterscheidung zwischen Umgangssprachen und Dialekten ist zwar pragmatisch brauchbar und entspricht wohl auch der intuitiven Selbsteinschätzung vieler Sprecher; sie ist aber linguistisch nicht zu begründen. Unterhalb der Standardsprache liegt ein Kontinuum von Sprachvarianten und Variantenkombinationen zwischen Basisdialekt und gehobener Umgangssprache (regionaler Standard), das man natürlich durch Zäsuren zu gliedern versuchen kann (Basisdialekt, Verkehrsdialekt usw.). Die Unterscheidungen stützen sich aber immer nur auf konkrete Sprachmerkmale und haben Gültigkeit immer nur für konkrete Sprachverhältnisse. Allgemein anwendbare Kriterien für eine Binnengliederung des Substandards gibt es nicht. Ich treffe mich in dieser Beurteilung mit Hermann Scheuringer (in diesem Band S. 332ff.). Fast alle Sprecher des BayerischÖsterreichischen verfügen über mehrere Register, deren Gebrauch durch die soziale Zugehörigkeit der Sprecher, durch Situation, Gesprächspartner, Redethema und Ausdrucksabsicht gesteuert wird. Diglossie, d.h. eine relativ strikte Verteilung von Dialekt und Standard auf bestimmte Domänen (im wesentlichen informell-formell) besteht in Südtirol und in einigen altertümlichen südbairischen Dialektgebieten. Dort haben sich auch die alten Ortsdialekte $\mathbf{z}$.T. noch recht ungebrochen erhalten. $\mathrm{Zu}$ - 
sammenfassend möchte ich zum Thema "Verfall" feststellen, daß das Ergebnis der Abbauvorgänge in bayer.-österr. Dialekten wiederum Dialekte oder dialektale Umgangssprachen sind, die die Funktionen von Dialekten weitgehend wahrnehmen können. Das mag für Sprecher der jüngsten Generation in München (so Kurt Rein in der Diskussion), unter etwas anderen Bedingungen auch in Wien nicht mehr zutreffen.

Bei der Themenfrage nach der Mundartrenaissance haben die Organisatoren der Tagung wohl auch - wie bei Verfall - eher an die metaphorische Bedeutung des Wortes gedacht, an Wiedererstarken oder an Expansion des Dialektgebrauches. Auch die Frage nach den Wirkungen oder dem Weiterleben der sogenannten Dialektwelle der 70er-Jahre mag mitgespielt haben.

Die Dialektwelle war in erster Linie ein Phänomen der neueren antitraditionellen Dialektliteratur und eine Folge der Sprachbarrierendiskussion, der 68er-Bewegung und der Wiederentdeckung der Region, der Provinz. Dialekt wurde (mif-)verstanden als emanzipatorische Sprache. Aber natürlich trägt Dialektgebrauch per se nichts bei zur Befreiung von obrigkeitlichen Zwängen (in ganz anderen Zusammenhängen stand die Entdeckung des Dialekts durch die Autoren der Wiener Gruppe 20 Jahre zuvor: für sie war der Dialekt ein neues Material zur Erreichung neuer ästhetischer Qualitäten). Auf das Sprachverhaiten der breiten Masse der Dialektsprecher im Süden hat diese Dialektwelle m.E. so gut wie keinen EinfluB gehabt. Am ehesten mag noch der Dialektgebrauch durch Liedermacher und Schlagersänger von Konstantin Wecker bis Hubert von Goisern Breitenwirkung insofern haben, als dadurch das sprachliche Selbstbewußtsein von Dialektsprechern bestätigt oder bestärkt wird.

Auf einer anderen Ebene ist es, nur in Österreich, doch zu einer Art von Mundartenrenaissance gekommen, wenn sie auch von den Sprechern weder als eine solche intendiert noch empfunden wird. Seit der Zäsur von 1945 oder noch deutlicher seit der Mitte der 50er-Jahre ist in Österreich die früher selbstverständliche Verbindlichkeit, in formellen Situationen Hochsprache $\mathrm{zu}$ verwenden, stark zurückgegangen. Im alltäglichen informellen Sprachgebrauch spielt die Standardsprache bestenfalls eine marginale Rolle. Weit überwiegend werden in den meisten Situationen verschiedene Varietäten von Umgangssprache verwendet. Nach einer 1984/85 durchgeführten Erhebung verteilt sich der durchschnittliche Alltagssprachgebrauch nach Selbsteinschätzung der Befragten zu $49 \%$ auf Dialekte, ebenfalls zu $49 \%$ auf Umgangssprachen und nur zu $2 \%$ auf die Standardsprache; in Mittel- und Grobstädten steigt der Anteil der Umgangssprache auf $63 \%$, in gehobenen Sozialschichten auf $74 \%$, der der Standardsprache jedoch nicht über 2-5\% (bei den über 
60 jährigen $6 \%$; Wiesinger 1992, S. 293f.). Die mittleren und erst recht die gehobenen, standardnahen Ausprägungen der Umgangssprache unterscheiden sich $\mathrm{zwar}$ deutlich von den ländlichen Basisdialekten, aber sie sind nicht frei von dialektalen Elementen. Als Friedrich Torburg 1951 aus dem Exil nach Wien zurückkehrte, hatte er den Eindruck, alle Leute redeten im Dialekt. Der Wiener Herbert Penzl, der seit 1934 in den Vereinigten Staaten lebte, beurteilte die Sprachsituation bei seinen vielen Österreichbesuchen nach dem 2. Weltkrieg ähnlich. Daraus folgert, $\mathrm{da \beta}$ die Bildungsschichten in den $20 / 30 \mathrm{er}-\mathrm{Jahren}$ sich sehr viel stärker um den Gebrauch einer korrekten Hochsprache bemühten als das seit den 50er-Jahren der Fall ist. Den Grund für diese Entwicklung sehe ich nicht nur in der allgemeinen Lockerung von Verhaltensnormen in vielen Bereichen (und natürlich nicht nur in Österreich), sondern vor allem in dem unbelasteten Verhältnis zur eigenen staatlichen Identität (anders als zur Zeit der 1. Republik 1918-1938). Daraus resultiert ein neues sprachliches SelbstbewuBtsein, das nicht mehr von dem ängstlichen Bemühen, „schön” zu sprechen und von dem schlechten Gewissen, es doch nicht richtig zu können, belastet ist. Der verminderte Druck, Standardsprache in bestimmten Situationen gebrauchen zu müssen, ist freilich der Fähigkeit, dies korrekt zu tun, nicht eben zuträglich. Als Reaktion auf die Erfahrungen der Jahre 1938-1945, Ansätze aus der Zeit des Ständestaates (1934-1938) weiterführend, fehlten in den ersten Jahren nach 1945 sprachisolationistische Tendenzen nicht völlig. Sie spielen heute kaum mehr eine Rolle. Die gelehrte und z.T. polemische Fachdiskussion über das österreichische Deutsch (vgl. zuletzt Muhr 1995) hat nach meiner Einschätzung mit dem sprachlichen Selbstverständnis der meisten Österreicher recht wenig zu tun. Vergleichbares zu dem „Förderverein Bayrische Sprache und Dialekte" gibt es in Österreich nicht. Die von diesem Verein vertretene anti- „preußische” Position kann offenbar in Bayern immer noch mit einer etwas breiteren Zustimmung rechnen.

In der Diskussion hat Klaus Mattheier bezweifelt, daß es im Süden Dialektverfall nicht gäbe. Jedenfalls erfolge ein Verlust an dialektaler "Tiefe" und die alten Ortsdialekte verschwänden weithin. Dies trifft sicher zu. Entscheidend ist aber, daran halte ich fest, daB an die Stelle der Ortsdialekte nicht etwa kolloquialer Standard tritt, sondern Varietäten, die weiterhin dialektal geprägt sind und zum Substandard gehören. Ob man sie Dialekte oder Umgangssprachen nennen mag, ist ein terminologisches, kein sachliches Problem. Auch die neuen Dialekte können Intimität schaffen und nach innen Identität ("Wir-Gefühl”), nach außen Abgrenzung vermitteln, wenn auch groBräumiger als früher. Aber auch die alten Dialekte waren ja nicht überall von Ort zu Ort verschieden, sondern von Dialektgebiet zu Dialektgebiet. 


\section{Literatur}

Ammon, Ulrich (1995): Die deutsche Sprache in Deutschland, Österreich und der Schweiz. Das Problem der nationalen Varietäten. Berlin/New York.

Muhr, Rudolf/Schrodt, Richard/Wiesinger, Peter (Hg.) (1995): Österreichisches Deutsch. Linguistische, sozialpsychologische und sprachpolitische Aspekte einer nationalen Variante des Deutschen. Wien.

Pollak, Wolfgang (1992): Was halten die Österreicher von ihrem Deutsch? Eine sprachpolitische und soziosemiotische Analyse der sprachlichen Identität der Österreicher. Wien.

Reiffenstein, Ingo (1976): Primāre und sekundäre Unterschiede zwischen Hochsprache und Mundart. Überlegungen zum Mundartabbau. In: Pohl, Dieter (Hg.): Opuscula slavica et linguistica. Klagenfurt. S. 337-347.

Reiffenstein, Ingo (1983): Deutsch in Ōsterreich. In: Reiffenstein, Ingo u.a. (Hg.): Tendenzen, Formen und Strukturen der deutschen Standardsprache nach 1945. Marburg. (Marburger Studien zur Germanistik 3). S. 15-27.

Reiffenstein, Ingo (1984): Standardsprache und Dialekt: Sprachnorm und Variation. In: Schulreport 1984/6, S. 1-2.

Wiesinger Peter (1992): Zur Interaktion von Dialekt und Standardsprache in Österreich. In: van Leuvensteijn, I. A./Berns, I.B. (Hg.): Dialect and Standard Language/ Dialekt und Standardsprache in the English, Dutch, German and Norwegian Areas. Amsterdam. (Koninklijke Nederlandse Akademie van Wetenschappen, Verhandelingen, Afd. Letterkunde, N. R. 150). S. 290-311. 